\title{
Demand Response Management Algorithm for Distributed Multi-Utility Environment in Smart Grid
}

\author{
L. PRIYA \\ Department of EEE \\ College of Engineering Guindy, \\ Anna University, Chennai \\ INDIA \\ V. GOMATHI \\ Department of EEE \\ College of Engineering Guindy, \\ Anna University, Chennai \\ INDIA
}

\begin{abstract}
Effective usage of Information and Communication Technologies (ICT) has started with a paradigm shift in the energy management and functioning of the conventional power grid. It also aids in the maintenance of the complete information about consumer usage pattern, power storage, supply and regulation. Blending of information and communication technologies with energy management creates a smart grid environment which makes it move to the next horizon. The smart grid environment, uplifts renewable energy sources and brings out novel strategies in the energy market. The new functioning of the energy market attracts more utility companies for decentralized power generation and optimizes the power price for the consumer. The consumer plays an active role in the demand response modelling to maximize the welfare of the utility and to obtain the optimized price for their demand. In this paper, a novel demand response management scheme is proposed for multi-utility environment. The utility companies function in a peer to peer manner to communicate effectively and to select a specific utility from a set of utilities for the power supply. The selection of single utility is based on a non-cooperative game theory algorithm where the demand and generated power should be balanced to maximize the welfare of the utility and the residential consumers. The power price can be updated in an equal interval to allow all the utilities to participate in the Distributed Multi-Utility Demand Response Management (DMDRM) system. The simulated results justify that the distributed noncooperative game theory algorithm certainly maximizes the welfare of the utility companies and residential consumers.
\end{abstract}

Key-Words: - demand response, smart grid, multi-utility, game theory, distributed environment, energy market

Received: July 6, 2020 Revised: September 18, 2020. Accepted: October 16, 2020. Published: October 31, 2020.

\section{Introduction}

Integrating Information and Communication Technologies with energy management will promote the power industries to bring different kind of strategies and techniques for the smart grid development and its applications. The existing traditional power grid networks does not meet the challenges in power management such as power quality, reliability, efficiency, ecology and economy. The traditional power grid systems need to be replaced with smart grids which support applications of the renewable energy, smart home and other smart services. In smart grid, the communication technologies extends the information services which will be used to understand the required power generation based on the demand. The smart grid system coordinates among the power generation, transmission, power distribution systems, smart metering and billing 
system. The bi-directional communication network enables the advanced metering infrastructure to exchange data such as power consumption, outage awareness and price update to the utility. Naturally wide range of security enforcement in communication and data availability are most important challenges in the smart grid [1].

Demand Response (DR) is one of the foremost feature in smart grid. This strategy is followed by the consumers to shift or reduce the consumption energy from peak hours. Demand response is defined as the changes in electric use by demandside resources from their normal consumption patterns to induce lower electricity use at times of high wholesale market prices or when system reliability is jeoparadized [2]. Different kind of demand response strategies are followed by the consumers under the incentive based program and price based program. To provide incentive based program to the consumers, the consumers have to shift their load from the peak period to off-peak period based on the results from the DR modelling. When the consumers shift their load to off-peak period, they are benefited by flexible power price at different time period of the day. So, when consumers participate in DR, there are three possible ways in which they can change their use of electricity $[3,4]$. Consumers limit their energy consumption through load curtailment strategies such as peak clipping, consumers shift their energy consumption to different time period of the day through load shifting or they consume power through onsite standby generated energy, thus limiting their dependence on the main grid through valley filling. In addition, to limit the peak demand of the residential consumers, demand response is achieved through the application of a variety of distributed resource types, including distributed generation, dispatchable load, storage and other resources that may contribute to modify the power supplied by the main grid. Traditionally power generation, transmission and distribution are functions of the power system. Electrical power industry has been dominated by a large utility that has a complete authority of all the activities in generation, transmission and distribution of power within its domain of operation. So, all the three functionalities are executed by the single utility.

Through the process of unbundling in deregulation, the task of traditional power system can open to competition wherever practical and profitable. The initial step of the restructuring process of the power industry has been the separation of the transmission activities from the electricity generation activities. Under the deregulation, the activities of vertically integrated utility has become competitive. Power generation has to compete in the competitive power generation market place. Transmission and Distribution have to operate as an open provider to deliver the services. The same competitiveness is also present in the retailing of the electricity at the consumer end. The consumer have the choice of selecting their utility based on utility trading. Even, the consumer can buy a portion of electricity from the first utility and remaining portion from the second utility. So, the demand response allows the residential consumers to reduce their billing tariff by selecting particular utility for buying the electricity. Consecutively, the utility is also benefitted through high pay-off in the competitive environment. This technology will ensure the power system more reliable, enhance the transparency and efficiency of the electricity market and lead to mutual financial benefits for both the power utility and consumers. In addition to reliability and efficiency, demand response strategies will reduce the generating emissions and alleviate the environmental impacts, by enabling a more efficient utilization of grid capacity. However, while consumers are benefited with lower electricity bills by shifting their load usage, they may be subject to some discomfort due to the shifting of their energy usage patterns.

In this paper, a distributed algorithm is designed among the utility companies which aims to maximize the welfare of the utilities and consumers. The competitiveness among the utilities are established in a distributed environment using the non- cooperative game theory algorithm.

\section{Literature Review}

Wide range of works have been carried out to model the demand response in smart grid environment. Most of the works are related to vertically integrated utility and the consumers. Li et al. [5] have proposed Demand Response approach for the utility company and the consumers to maximize the payoff. Datchanamoorthy et al. [6] have presented an algorithm for finding non-peak hours for the consumers and recommend them to shift their load from peak hours to non-peak hours in monopoly utility markets. Moreover, game theory has been applied to the DRM problem in smart grid since it is effective in dealing with complicated interaction. The residential consumers can shift their usage of shiftable appliances or reduce the power 
consumption of the non-shiftable appliances to reduce the tariff. This leads to discomfort among the consumers participating in the DR program. Ma et al. [7] have considered the discomfort of the consumers and designed an optimal scheduling strategy and achieved the desired trade-off between the utility and consumers in the single utility environment. Setlhaolo et al. [8] have developed a load scheduling of residential consumers using a mixed-integer nonlinear optimization model. The Time of Use (TOU) pricing method has been used in this model for about five time periods with varying prices. Kinhekar et al. [9] have proposed a DR procedure for load shifting using the integer genetic algorithm to benefit both the utility company and the consumer. This method attempted to fit the actual consumption power curves and the utility objective curve at each time period. The consumer information truthfulness is another important factor in cooperative load scheduling that are analyzed in many studies. Samadi et al. [10] have used the Vickrey Clarke Groves optimization mechanism for efficient energy consumption. According to the problem formulation, the payment of one consumer depends on the other consumer. Thus, consumers must be honest while declaring their load consumption information. Many authors have introduced the Internet of Things (IoT) for automating the demand response to control the appliances remotely. Ali et al. [11] have proposed a pricing policy framework for the DR in smart grid based IoT networks. According to the prices announced by the provider, the system controls the appliances of the user remotely during the peak hours of the day. Individual users adapt their energy consumption according to the price signals to maximize their own benefits. Chai et al. [12] have designed a two-level game model for demand response with multiple utilities. Among the utilities, a specific utility was selected based on the equilibrium and that utility will serve the power to the consumers. An evolutionary game was used for interaction among the residential consumers and non-cooperative game was used for interaction among utilities. Deng et al. [13] have determined the user's demand and utility supply through a distributed real-time demand response program. This model does not require any aggregator or a third party to monitor and control. Fan and Zhong [14] have designed a distributed demand response algorithm for novel charging PHEV in smart grid.
Maharjan et al. [15] have proposed a Stackelberg game where each utility company and each user intend to maximize their own benefits. This approach requires coordination among residential consumers and utility companies. Since, this approach is cooperative, it is not practically realistic.

In case of single utility company in the smart grid environment, it is the responsibility of that particular utility company to meet the demand of the residential consumers. On the other hand, in case of multi-utility companies, there exist a competition among them and all the utility companies are responsible to meet the demand. In this paper, a distributed multi-utility demand response management algorithm has been proposed in the multi-utility environment using the non-cooperative game theory for the selection of a utility among the set of utility companies.

\section{Distributed Multi-utility Demand Response management (DMDRM) model}

In this proposed model, a Demand Response Management (DRM) system is designed for multiple utility companies and multiple residential consumers with different category of loads. The relationship among the utilities is modelled as a non-cooperative game theory algorithm, which runs in a distributed environment. Every utility company decides its price and generation of power based on the demand of the residential consumers. Each residential consumer coordinates the power demand based on the price decided by the utility company. The advantage of the multi-utility companies in the system ensures more flexible and lower prices for the residential consumers, a wide range of tariff choices and also maintains the balance between the supply and the demand.

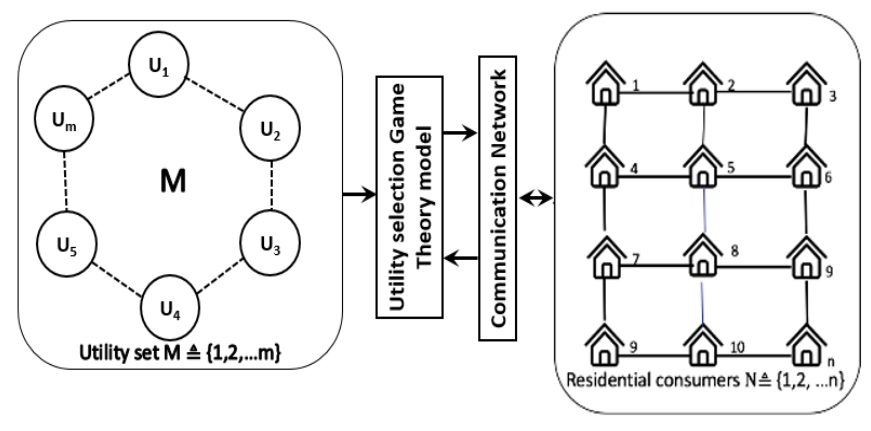

Fig. 1 : Analytical Framework for Distributed DRM model 
The figure 1 shows the analytical framework for the Distributed Demand Response Management model of a smart distribution power grid with multiple utility companies and load subscribers or residential users. In the analytical framework, all the utility companies are connected through a communication network as a diagraph. The diagraph $\mathrm{G}$ is a ring consisting of $\mathrm{M}$ utility companies, numbered from 1 to $\mathrm{m}$ in the clockwise direction. The requirement of the game is that, exactly one utility company should be selected as a leader who is offering the attractive price to the residential consumers for the power supply. The number of utility company in the network can either be known or unknown to the residential users.

\section{System Model}

In the smart grid environment, each residential consumer is assumed to be equipped with an energy consumption scheduler embedded in the smart meter. The integrated scheduler module in the smart meter schedules and controls the energy consumption of the residential user. It also coordinates each user with other user and also with the utility company. Let the smart distribution grid consists of a set $N \triangleq\{1,2, \ldots . n\}$ of users and $M \triangleq$ $\{1,2, \ldots . m\}$ utility companies. The residential users are connected to the utility companies through a communication network. The intended time period for the usage of the appliances by the user in a day is divided into $\mathrm{H}$ time slot where $H=\{1,2, \ldots . h\}$.

\subsection{Energy Cost model and welfare of the utility company}

The total power generation of the utility company $m \in M$ at time $h \in H$ is defined as $l_{h}^{m}$ and the cost function of the utility company $m$ at time slot $h$ is $C_{h}^{m}\left(l_{h}^{m}\right)$ which is assumed to be increasing and strictly convex. The quadratic function without loss of generality is $[10,12,17]$

$$
C_{h}^{m}\left(l_{h}^{m}\right)=a_{h}^{m}\left(l_{h}^{m}\right)^{2}+b_{h}^{m} l_{h}^{m}+c_{h}^{m}
$$

Where $a_{h}^{m}>0$ and $b_{h}^{m}, c_{h}^{m} \geq 0$ are constant parameters. From micro economics [19] the concept of utility function is used to model the different price scenarios. Having various options of utility function, the behavior of different users can be modelled [17]. The supply capacity of the utility company should be greater than its power generation. Let $l_{\max }^{m}$ denote the power supply capacity of the utility. Thus, the power supply of the utility is bounded by the constraint

$0 \leq l_{h}^{m} \leq l_{\max }^{m}$

Let $l_{n, h}^{m}$ represents the power generation of utility company to $n$ users at time slot $h$ and $S_{n, h}^{m}$ be the amount of power sold from utility company $m$ to user $n$. If $D_{n, h}^{m}$ is the power demand of the user $n$ from a utility company $m$ and $P_{n, h}^{m}$ denotes the power price set by the utility company then

$S_{n, h}^{m}=\min \left(l_{n, h}^{m}, D_{n, h}^{m}\right)$

Hence, the welfare of the utility company is defined as the difference between the profit of all its sold power to the residential users and the cost of the power generated by the utility. Therefore, the welfare function of the utility company $\mathrm{m}$ for the user $\mathrm{n}$ at time slot $\mathrm{h}$ is expressed as

$U_{h}^{m}\left(P_{n, h}^{m}, S_{n, h}^{m}\right)=\sum_{n=1}^{N} P_{n, h}^{m} S_{n, h}^{m}-C_{h}^{m}\left(l_{h}^{m}\right)$

\subsection{Utility and Welfare function of the Residential user}

The residential users demand behavior varies from time to time depending on the requirement such behavior of different users can be modelled through different choices of utility. The residential users are assumed to act independently without any interaction with each other, but they are more concerned about the power prices from the utility company. On the residential user side, let $x_{n, h}^{m}$ denotes the total energy demand of the user $n$ from utility company $m$ at time slot $h$. The energy demand is bounded by

$\sum_{m \in M} x_{n, h}^{m} \geq b_{n}^{h} \quad \forall n \in N, \quad \forall h \in H$

where $b_{n}^{h}$ is the baseline demand of the user $n$ at time slot $h$, in addition to the elastic demand $\mathrm{e}_{\mathrm{n}}$ of the user $n$. The baseline and elastic demand requirement are considered to form a user demand constraint

$$
\sum_{h \in H} \sum_{m \in M} x_{n, h}^{m} \geq \sum_{h \in H} b_{n}^{h}+e_{n} \quad \forall n \in N
$$

The utility function of the residential user should be 
non-decreasing concave and differentiable [12] which indicates that the demand satisfaction is directly proportional to the user satisfaction. This paper considers quadratic utility function corresponding to linear decreasing marginal benefit $[10,12,17]$ which is given as,

$u_{n, h}\left(x_{n, h}\right)=\left\{\begin{array}{cc}v_{n, h} x_{n, h}-\frac{\alpha_{n, h}}{2} x_{n, h}^{2}, \text { if } 0 \leq x_{n, h} \leq \frac{v_{n, h}}{\alpha_{n, h}} \\ \frac{v_{n, h}}{\alpha_{n, h}}, & \text { if } x_{n, h} \geq \frac{v_{n, h}}{\alpha_{n, h}}\end{array}\right.$

where $v_{n, h}$ and $\alpha_{n, h}$ are predetermined time varying parameters. These parameters clearly picturize the variation in power demand of different residential users at different time slot. The larger $v_{n, h}$ and smaller $\alpha_{n, h}$ leads to increased power demand as the users' main objective is to maximize its utility.

If a residential user $n$, consumes $x_{n, h}^{m}$ amount of power supply during time $h$ from the utility company $m$ at a price of $p_{n, h}^{m}$ then it is charged as

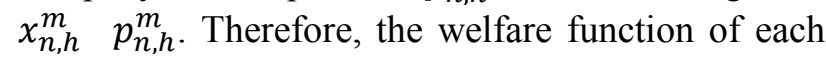
user $\mathrm{n}$ can be described as

$w_{n, h}\left(x_{n, h}^{m}\right)=u_{n, h}\left(x_{n, h}^{m}\right)-\sum_{m=1}^{M} p_{n, h}^{m} x_{n, h}^{m}$

subject to

$x_{n, h \text { min }} \leq x_{n, h} \leq x_{n, h \max }$

In the equation (9), $x_{n, h \text { min }}$ is the power consumed by non-shiftable appliances of the residential user and $x_{n, h \max }$ is the power consumed by all the appliances of the residential consumer.

\subsection{Utility and Consumer Interaction}

The need for electricity varies between each user depending on their consumption pattern. Consider, there are multiple utility companies with different power prices. The consumption charge of each user depends on the selection of the particular utility and the per unit price, set by that utility. The main objective of the residential user is to procure optimal amount of power at lower price to maximize its own benefit. On the other hand, from the utility point of view, they always plan to sell the optimal power at higher price to achieve its welfare. In this regard, appropriate strategies should be implemented on the utility and user side to maintain the balance between the supply and demand. The utility companies set and communicate the per unit power price to the users. The amount of power to be purchased by the user is based on the price information received from the utilities. The optimization problem of the user can be defined as

$\operatorname{Maximize}\left(w_{n, h}\left(x_{n, h}\right)\right)$,

subject to the equation (9).

The optimization problem is a concave maximization problem which can be optimized using convex programming technique such as interior point method or efficient set method [20]. The solution of the equation (10), can be defined as

$x_{n, h}^{m}=\left\{\begin{array}{rr}x_{n, h}^{m} & \text { if } m=\arg \min \left(p_{n, h}^{m}\right) \\ 0, & \text { otherwise }\end{array}\right.$

where $x_{n, h}^{m}$ is the optimal demand of the user $n$ from the utility $\mathrm{m}$ during time $h$. It is clear from the results that the users' choice is the utility company who provide the power at the lowest price as far as they supply sufficient power. At the time slot $h$, the probability of user $\mathrm{n}$ choosing the utility $\mathrm{m}$ be $y_{n, h}^{m}$. If the utility supplies enough power to the user then $y_{n, h}^{m}$ can be described as $0 \leq y_{n, h}^{m} \leq 1$.

According to the equation (8) and (9), the optimal power consumption of the user $n$ buying power from the utility $m$ at time $\mathrm{h}$ can be obtained by equating the derivatives of equation (8) with respect to $x_{n, h}^{m}$ to zero.

$x_{n, h}^{m}=\arg \max w_{n, h}^{m}\left(x_{n, h}^{m}\right)$

equation (8) can be simplified as

$$
\begin{aligned}
& w_{n, h}\left(x_{n, h}^{m}\right)=u_{n, h}\left(x_{n, h}^{m}\right)-p_{n, h}^{m} x_{n, h}^{m} \\
& \frac{\partial w_{n, h}}{\partial x_{n, h}^{m}}=\frac{\partial\left(u_{n, h}\left(x_{n, h}^{m}\right)\right)}{\partial x_{n, h}^{m}}-p_{n, h}^{m}
\end{aligned}
$$

Using equation (7) in equation (14)

$$
\begin{aligned}
& =\frac{\partial\left(v_{n, h} x_{n, h}-\frac{\alpha_{n, h}}{2} x_{n, h}^{2}\right)}{\partial x_{n, h}^{m}}-p_{n, h}^{m} \\
& v_{n, h}-\alpha_{n, h} x_{n, h}-p_{n, h}^{m}=0
\end{aligned}
$$




$$
\begin{aligned}
& x_{n, h} \quad \alpha_{n, h}=v_{n, h}-p_{n, h}^{m} \\
& x_{n, h}=\frac{v_{n, h}-p_{n, h}^{m}}{\alpha_{n, h}}
\end{aligned}
$$

Therefore,

$$
x_{n, h}^{m}=\left\{\begin{array}{c}
x_{n, h \text { min },} \quad \text { if } \frac{v_{n, h}-p_{n, h}^{m}}{\alpha_{n, h}}<x_{n, h \text { min }} \\
\frac{v_{n, h}-p_{n, h}^{m}}{\alpha_{n, h}}, \text { if } x_{n, h \text { min }} \leq \frac{v_{n, h}-p_{n, h}^{m}}{\alpha_{n, h}}<x_{n, h \max } \\
x_{n, h \text { min },} \text { if } \quad x_{n, h \max }<\frac{v_{n, h}-p_{n, h}^{m}}{\alpha_{n, h}}
\end{array}\right.
$$

The user cannot get sufficient power from the single utility due to the restriction on the power generation capacity. In those situations, the user may get $x_{n, h}^{m}$ amount of power from the utility company whose price is the lowest and then buy the remaining amount of power from the utility company which offers the next lowest price. In this way, the optimal power demand can be calculated for utilities using the problem formulation given above.

\subsection{Non - Cooperative game modelling for utilities}

In the multi-utility environment, utility company will act first and then the user acknowledge on the basis of the power price. Since the two actions are in sequence the interaction between the utility company and residential users are modelled using game theory. Each utility makes their own decision and want to maximize their profit. Therefore, each utility has to compete with other utility to attract more residential users which is modelled as a non cooperative game. So, the game is to maximize the profit by competing with other utility companies and attract more consumers.

In the game, nodes in the ring are utility companies referred to as players. The strategy of the game is to select a utility from the set of utilities. The payoff of the game is welfare of the utility company.

Therefore, the welfare function of the utility is formulated using equation (1) in equation (4)

$U_{h}^{m}=P_{n, h}^{m} S_{n, h}^{m}-\left[a_{h}^{m}\left(l_{h}^{m}\right)^{2}+b_{h}^{m} l_{h}^{m}+c_{h}^{m}\right]$
$U_{h}^{m}=P_{n, h}^{m} S_{n, h}^{m}-a_{h}^{m}\left(l_{h}^{m}\right)^{2}-b_{h}^{m} l_{h}^{m}-c_{h}^{m}$

As introduced in $[4][11][16]$ equation (19) is reduced to
$U_{h}^{m}= \begin{cases}P_{n, h}^{m} S_{n, h}^{m}-\left[a_{h}^{m}\left(l_{h}^{m}\right)^{2}+b_{h}^{m} l_{h}^{m}+c_{h}^{m}\right], & \text { if } r_{n, h}^{m} \geq 0 \\ P_{n, h}^{m} D_{n, h}^{m}-\left[a_{h}^{m}\left(l_{h}^{m}\right)^{2}+b_{h}^{m} l_{h}^{m}+c_{h}^{m}\right], & \text { Otherwise }\end{cases}$

where $r_{n, h}^{m}$ denotes the difference between demand and power generation. That is

$r_{n, h}^{m}=D_{n, h}^{m}-l_{n, h}^{m}$

When $r_{n, h}^{m} \geq 0$, the power demand of the $n^{\text {th }}$ user from the $m^{\text {th }}$ utility company is greater than the power generation. So, the generated power can be sold out to the residential users, otherwise the generated power cannot be sold out. In such case, the utility company changes the strategy by reducing the power price to increase the user's demand. To maintain the reliability of the system, the power balance equation must be satisfied. Power generated by the utility company should be within the limits which is defined as

$$
x_{n, h \min }^{m} \leq l_{n, h}^{m} \leq x_{n, h \max }^{m}
$$

As two different variables such as power price and generation $\left(P_{n, h}^{m} \& l_{n, h}^{m}\right)$ are involved in equation (20) which cannot be solved by any direct method. So, it is assumed that the power price is known and the optimal power generation is calculated as follows

$$
\begin{aligned}
& \operatorname{Maximize}\left(U_{h}^{m}\right) \text { subject to } \\
& l_{n, h}^{m} \leq l_{\max }^{m} \text { and equation (22) }
\end{aligned}
$$

The equation (23) is a concave maximization problem, which has to be optimized. The results depict that the utility company supplies power to the residential user with highest demand to maximize its own welfare. The updated power price can be calculated based on equation (24).

$p_{n, h}^{m}(i+1)=p_{n, h}^{m}(i)+\theta_{m} r_{n, h}^{m}(i)$

where $\theta_{m}$ is a constant which subject to increment size $\mathrm{i}$. The proper price set by the utility is based on the balance between power demand and generation.

\subsection{Algorithm}

In a distributed environment,

1. Each utility $m$ is identified by a Unique Identifier (UID), chosen from ordered space of identifier. 
2. All the active utilities store the generated power $l_{n, h}^{m}$ and set the price by satisfying $\mathrm{r}_{\mathrm{n}, \mathrm{h}}^{\mathrm{m}} \geq 0$.

3. Utilities are arranged in such a way that unit price $p_{n, h}^{m}$ set by the utility. The algorithm flows, as the problem of ensuing that only one utility at a time to supply the demand. The intension is that in any distributed environment, utilities in a communication system may need to agree on whether or not it has been selected for supplying the power to the residential consumers.

4. To ensure fairness, all utilities update their prices in steps at every hour. The utility with the highest quoted price at a given hour then brings it down to the lowest quote for the subsequent hour.

5. The user with the high priority gets the supply at each supply band followed by the user with the medium and low priority while demand satisfied. If $D_{n, h}^{m}-l_{n, h}^{m}<0$, demand is not met by one utility, it buys from the next utility from the ordered dataset obtained.

6. Every action is communicated to other utilities to maintain the transparency in the system in a distributed environment.

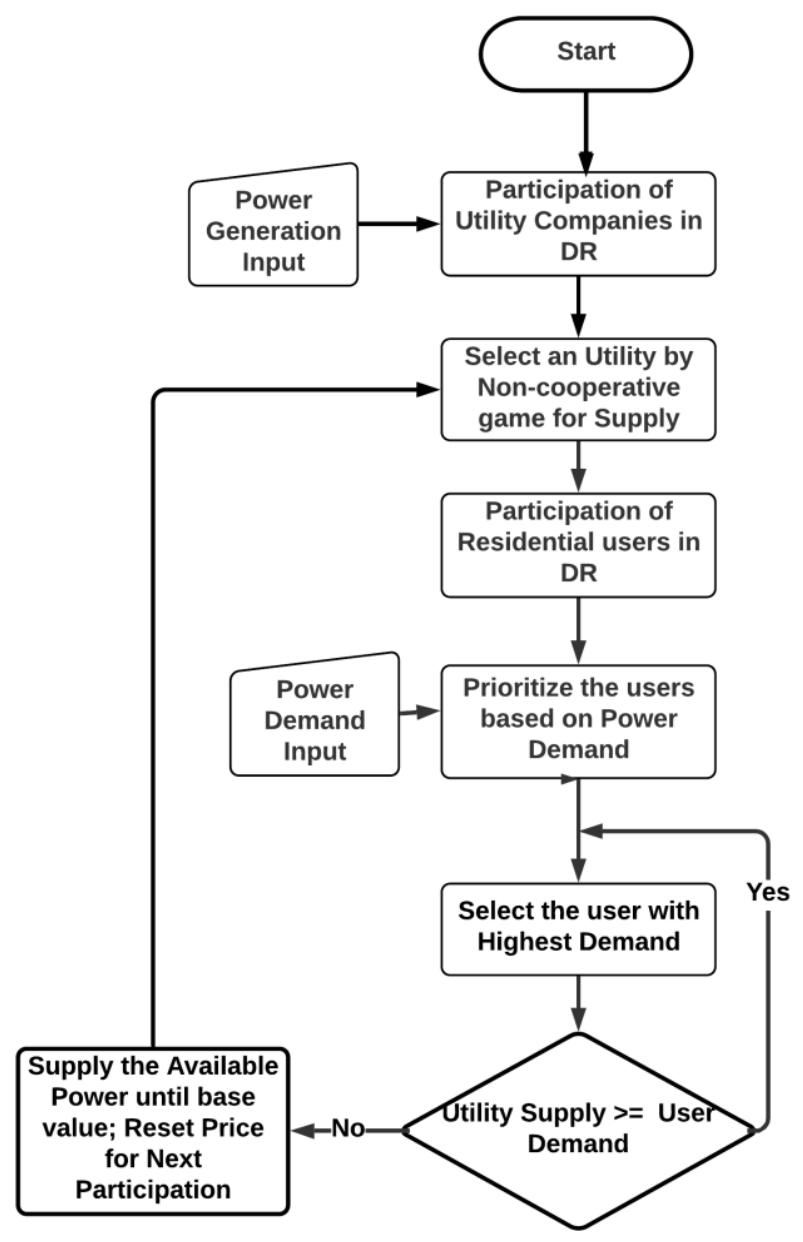

Fig 3: Flowchart for DMDRM

This algorithm benefits the residential consumer by offering low prices for their demand and also maximize the welfare of the utility companies to sell out their generated power. The algorithm is simulated with a sample data to check the complexity and ensure the setting to reach the equilibrium state of the non-cooperative game.

\section{Results and Discussion}

To simulate the distributed multi-utility demand response management algorithm, five residential users where every user has multiple shiftable and non-shiftable loads and three utilities have been considered. The demand of the residential consumers as well as the power generation of the utility companies are considered for eight hours. The residential consumers are having two kind of loads namely baseline load and shiftable load. Both the loads are considered for the verification of the proposed system. The following table 1 shows that the total power demand of the residential consumers for eight hours

Table 1: Power Demand of 5 Users

\begin{tabular}{|l|l|c|}
\hline Sl.no & User details & $\begin{array}{c}\text { Power Demand in } \\
\text { Watts }\end{array}$ \\
\hline 1 & User-1 & 5120 \\
\hline 2 & User-2 & 3260 \\
\hline 3 & User-3 & 2580 \\
\hline 4 & User-4 & 3940 \\
\hline 5 & User-5 & 2140 \\
\hline
\end{tabular}

The following table 2 shows the power generated by the three utilities for eight hours. According to the proposed system, the demand profile of the residential consumers are estimated.

Table 2: Power Generation of three utilities

\begin{tabular}{|l|l|c|}
\hline Sl.no & \multicolumn{1}{|c|}{$\begin{array}{c}\text { Utility } \\
\text { details }\end{array}$} & $\begin{array}{c}\text { Power Demand in } \\
\text { Watts }\end{array}$ \\
\hline 1 & Utility-1 & 5270 \\
\hline 2 & Utility -2 & 6510 \\
\hline 3 & Utility -3 & 5260 \\
\hline
\end{tabular}

The estimated power profile of each consumer respective to 8 hours is shown in table 3 .

Table 3 : Power demand profile of the consumers in Watts

\begin{tabular}{|l|l|l|l|l|l|l|l|l|}
\hline Hour & $\mathbf{1}$ & $\mathbf{2}$ & $\mathbf{3}$ & $\mathbf{4}$ & $\mathbf{5}$ & $\mathbf{6}$ & $\mathbf{7}$ & $\mathbf{8}$ \\
\hline
\end{tabular}




\begin{tabular}{|c|r|r|r|r|r|r|r|r|}
\hline Users & & & & & & & & \\
\hline $\mathbf{1}$ & 80 & 80 & 1080 & 1080 & 1080 & 40 & 840 & 840 \\
\hline $\mathbf{2}$ & 870 & 870 & 120 & 80 & 80 & 80 & 80 & 1080 \\
\hline $\mathbf{3}$ & 390 & 390 & 390 & 40 & 80 & 430 & 430 & 430 \\
\hline $\mathbf{4}$ & 790 & 830 & 40 & 80 & 80 & 40 & 1040 & 1040 \\
\hline $\mathbf{5}$ & 80 & 80 & 40 & 40 & 790 & 870 & 120 & 120 \\
\hline
\end{tabular}

According to the algorithm, each utility sets its power price independently for their generated power as non-cooperative game and the price can be updated in subsequent hours. The initial setting and updating of price by one utility does not affect the other utility decision. The utility which sets its price as the lowest is selected for the supply to the residential consumer who is having high priority based on the required demand. The table 4 depicts the power generated by the utilities with respective to each hour. Since, the electricity demand of the residential consumer for each hour is estimated, the intension of the game theory algorithm is to sell out the generated power to the residential consumer.

TABLE 4: Utility Power generation in Watts

\begin{tabular}{|c|c|c|c|c|c|c|c|c|}
\hline \multicolumn{1}{|c|}{ Hour } & $\mathbf{1}$ & $\mathbf{2}$ & $\mathbf{3}$ & $\mathbf{4}$ & $\mathbf{5}$ & $\mathbf{6}$ & $\mathbf{7}$ & $\mathbf{8}$ \\
\hline $\mathbf{1}$ & 1000 & 800 & 900 & 500 & 110 & 460 & 1050 & 800 \\
\hline $\mathbf{2}$ & 800 & 450 & 400 & 540 & 1000 & 520 & 850 & 1400 \\
\hline $\mathbf{3}$ & 410 & 1000 & 370 & 280 & 1000 & 480 & 610 & 1310 \\
\hline
\end{tabular}

If the utility does not meet the required demand of the residential consumer, then the consumer will purchase the remaining power from the next utility who is having the lowest price. For the next hour, the price will be updated at the utility side to allow all the utilities to participate in the game. The loop will be continued until the residential consumer demand is met by the utility companies. Since, the power generation is based on the estimated power demand, the utility welfare is also maximized.

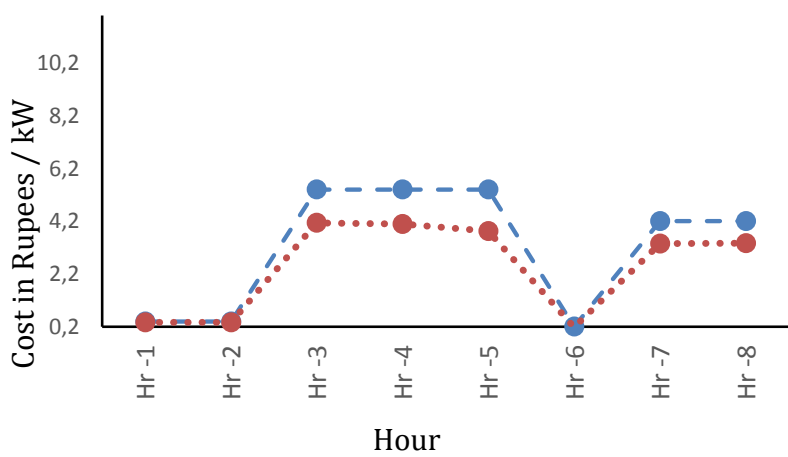

— - User-1 (Single Utility) ...... User-1 (Multi-Utility)

Fig. 4: Comparison of cost benefit with single and multi-utility for user-1

The figure 4 shows the user- 1 benefit in the multiutility environment. For the hour 1, 2 and 6 , the demand of the user- 1 is 80,80 and 40 watts respectively as in table 1 . Since, the power demand is very low, there is not much difference compared to single utility and multi-utility, but for the remaining hours the user-1 is benefited considerably with multi-utilities. Figure 5 depicts the cost difference between the single and multi-utility environment for user-2. Here, eight hours have been considered with different loads at the residential side. The lowest load starts from 40 watts. In the normal environment such as 24 hours with different loads, there will be vast difference between single utility and multi-utility.

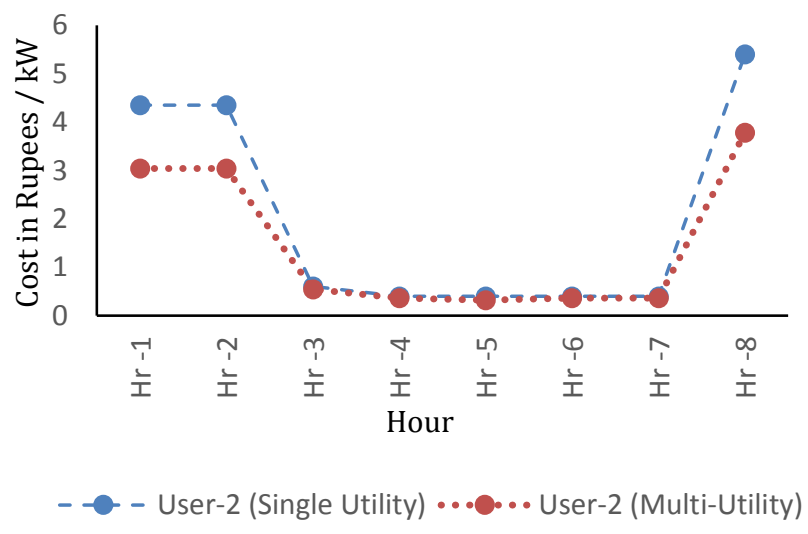

Fig. 5 : Comparison of cost benefit with single and multi-utility for user-2

The figure 6 shows the cost benefit comparison of user- 3 with single utility and multi-utility. Hour -1 , $2,3,6,7$ and 8 justifies that there is a vast difference 
in the demand in the multi-utility environment.

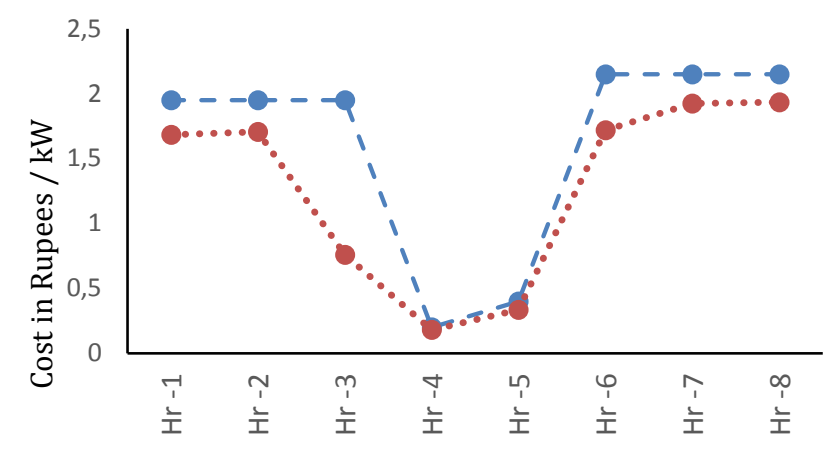

- ๑- User-3 (Single Utility) ...... User-3 (Multi-Utility)

Fig. 6 : Comparison of cost benefit with single and multi-utility for user-3

The figure 7 and 8 illustrates, the cost benefit for the user 4 and user 5 in the multi-utility environment respectively. As defined in the algorithm, the purchase of the power started from a utility which offers lowest price. If the demand is not met then the consumer will be supplied from the next utility who is offering the second lowest price. So, the consumer will purchase power only when the demand and generation are balanced.

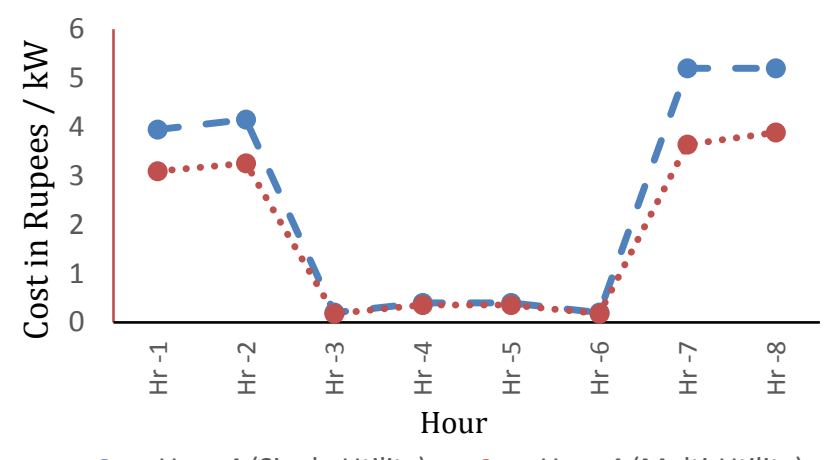

Fig. 7 : Comparison of cost benefit with single and multi-utility for user-4

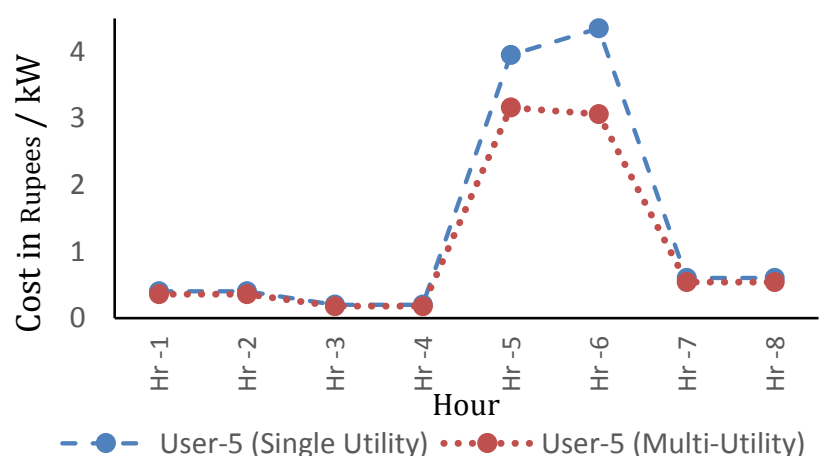
reshaped the electricity market to benefit the residential consumer and to improve the performance of the smart grid. The proposed distributed demand response management modelling in multi-utility environment has been implemented through non-cooperative game theory algorithm. The utility companies are coherently functioning in a distributed atmosphere and maximize the welfare of themselves as well as the consumer. The simulated results show that the multi-utility electricity market benefits the consumer and maximizes the welfare of all the utilities participating in the game. The distributed demand response modelling algorithm can be extended to PHEV charging stations in the smart grid and the excess renewable energy generated by the consumers can be sold out to the utilities.

The figure 9 shows the overall benefit of the hour. The comparison shows that the multi-utility environment is more beneficial to the residential consumers than the single utility environment.

The participated utilities in the distributed demand response management modelling sell out all their In addition, no utility is benefited maximum, when compared to other utility. So, equal benefit among all the utilities. All the utilities vary their price independently in the non-cooperative game and reach their equilibrium.

\section{Conclusion and Future Work}




\section{References:}

[1] Pinson, Pierre, and Henrik Madsen. "Benefits and challenges of electrical demand response: A critical review." Renewable and Sustainable Energy Reviews 39 (2014): 686-699.

[2] K.Ma, G.Hu, and C.J.Spanos, "Distributed energy consumption control via real-time pricing feedback in smart grid," IEEE Trans. Control Syst. Technol., vol. 22, no. 5, (2014), pp. 1907-1914.

[3] Mohagheghi S, Stoupis J, Zhenyuan Wang, Zhao Li, Kazemzadeh H. Demand response architecture: integration into the distribution management system. In: Proceedings of the first IEEE international conference on smart grid communications (Smart Grid Communication). 2010; p. 501-6.

[4] Deng, Ruilong, Zaiyue Yang, Mo-Yuen Chow, and Jiming Chen. "A survey on demand response in smart grids: Mathematical models and approaches." IEEE Transactions on Industrial Informatics 11, no. 3 (2015): 570582.

[5] Li, Na, Lijun Chen, and Steven H. Low. "Optimal demand response based on utility maximization in power networks." In 2011 IEEE power and energy society general meeting, pp. 1-8. IEEE, 2011

[6] S. Datchanamoorthy, S. Kumar, Y. Ozturk, and G. Lee, "Optimal time-of-use pricing for residential load control," in Proc. IEEE SmartGrid Communication, 2011, pp. 375-380.

[7] Ma, Kai, Ting Yao, Jie Yang, and Xinping Guan. "Residential power scheduling for demand response in smart grid." International Journal of Electrical Power \& Energy Systems 78 (2016): 320-325

[8] Setlhaolo D, Xia X, Zhang J. Optimal scheduling of household appliances for demand response. Electric Power System Research 2014; vol:116: pg 24-8.

[9] Kinhekar N, Padhy NP, Gupta HO. Multi objective demand side management solutions for utilities with peak demand deficit. International Journal of Electrical and Power Energy systems Vol.55, pg: 612-619, 2014

[10] Samadi P. Advanced demand side management for the future smart grid using mechanism design. IEEE Transactions in Smart Grid 2012;Vol 3; pg:1170-80.
[11] Ali S, Ahmad R, Kim D. A study of pricing policy for demand response of home appliances in smart grid based on M2M. In Proceedings of $201210^{\text {th }}$ international conference on frontiers of information technology; 2012. p.231-236.

[12] Chai, Bo, Jiming Chen, Zaiyue Yang, and Yan Zhang. "Demand response management with multiple utility companies: A two-level game approach." IEEE Transactions on Smart Grid 5, no. 2 (2014): 722-731.

[13] Deng, Ruilong, Zaiyue Yang, Fen Hou, MoYuen Chow, and Jiming Chen. "Distributed real-time demand response in multisellermultibuyer smart distribution grid." IEEE Transactions on Power Systems 30, no. 5 (2015): 2364-2374.

[14] Fan, Zhong. "A distributed demand response algorithm and its application to PHEV charging in smart grids." IEEE Transactions on Smart Grid 3, no. 3 (2012): 1280-1290.

[15] S.Maharjan, Q.Zhu, Y.Zhang, S.Gjessing, and T.Basar,"Dependable demand response management in the smart grid: A Stackelberg game approach," IEEE Trans. Smart Grid, vol. 4, no. 1, pp. 120-132, Jan. 2013.

[16] Chen, Lijun, Na Li, Steven H. Low, and John C. Doyle. "Two market models for demand response in power networks." In 2010 First IEEE International Conference on Smart Grid Communications, pp. 397-402. IEEE, 2010.

[17] Samadi, Pedram, Amir-Hamed Mohsenian-Rad, Robert Schober, Vincent WS Wong, and Juri Jatskevich. "Optimal real-time pricing algorithm based on utility maximization for smart grid." In 2010 First IEEE International Conference on Smart Grid Communications, pp. 415-420. IEEE, 2010.

[18] M. Fahrioglu and F. Alvarado, "Using utility information to calibrate customer demand management behavior models," IEEE Trans. on Power Systems, vol. 16, no. 2, pp. 317-322, May 2001

[19] S. Boyd and L. Vandenberghe, Convex Optimization. University Press, 2004. Cambridge.

[20] A. Mas-Colell, M. D. Whinston, and J. R. Green, Microeconomic Theory, 1st ed. USA: Oxford University Press, 1995.

\section{Creative Commons Attribution License 4.0 (Attribution 4.0 International, CC BY 4.0)}

This article is published under the terms of the Creative Commons Attribution License 4.0

https://creativecommons.org/licenses/by/4.0/deed.en_US 